\title{
Image Filtering Based on GMSK
}

\author{
Sehba Yousuf ${ }^{1}$, Er. Arushi Baradwaj ${ }^{2}$ \\ ${ }^{1}$ M.Tech Scholar, ${ }^{2}$ Assistant Professor \\ Department of Electronics and Communication Engineering, \\ Sri Sai College of Engineering \& Technology Badhani, Pathankot, Punjab, India
}

\begin{abstract}
Image Denoising is an important pre-processing task which is used before further processing of image. The purpose of denoising is to remove the noise while retaining the edges and other detailed features. This noise gets introduced during the process of acquisition, transmission \& reception and storage \& retrieval of the data. Due to this there is degradation in visual quality of image. The noises which are of major considerations are Additive White Gaussian Noise (AWGN) and Impulsive Noise.
\end{abstract}

\section{Keyword: Denoising, AWGN, MSE, SPN}

\section{INTRODUCTION}

Image processing is a method to perform some operations on an image, in order to get an enhanced image or to extract some useful information from it. It is a type of signal processing in which input is an image and output may be image or characteristics/features associated with that image. Nowadays, image processing is among rapidly growing technologies. It forms core research area within engineering and computer science disciplines too.

Image processing basically includes the following three steps:

Importing the image via image acquisition tools.

Analyzing and manipulating the image.

Output in which result can be altered image or report that is based on image analysis.

There are two types of methods used for image processing namely, analogue and digital image processing. Analogue image processing can be used for the hard copies like printouts and photographs. Image analysts use various fundamentals of interpretation while using these visual techniques. Digital image processing techniques help in manipulation of the digital images by using computers. The three general phases that all types of data have to undergo while using digital technique are pre-processing, enhancement, and display, information extraction. Image denoising is one of the important and essential components of image processing. Many scientific data sets picked by the sensors are normally contaminated by noise. It is contaminated either due to the data acquisition process, or due to naturally occurring phenomenon. There are several special cases of distortion. One 2 of the most prevalent cases is due to the additive white Gaussian noise caused by poor image acquisition or by communicating the image data through noisy channels. Other categories include impulse and speckle noises. The goal of denoising algorithm is to remove the unwanted noise while preserving the important signal features as much as possible. Noise elimination introduce artefacts and blur in the images. So image denoising is still a challenging task for the investigators. Several methods are being developed to perform denoising of corrupted images. The two fundamental approaches of image denoising are the spatial filtering methods and transform domain filtering methods. Spatial filters operate a low-pass filtering on a set of pixel data with an assumption that the noise reside in the higher region of the frequency spectrum. Spatial low-pass filters not only provide smoothing but also blur edges in signals and images. Whereas high pass filters improve the spatial resolution, and can make edges sharper, but it will also intensify the noisy background. Fourier transform domain filters in signal processing involve a trade-off between the signal-to-noise ratio (SNR) and the 
spatial resolution of the signal processed. Using Fast Fourier Transform (FFT) the denoising method is basically a low pass filtering procedure, in which edges of the denoised image are not as sharp as it is in the original image. Due to FFT basis functions the edge information is extended across frequencies, which are not being localized in time or space. Hence low pass-filtering results in the spreading of the edges.

\section{PERFORMANCE EVALUATION IMAGE DENOISING}

Objective image quality measures play important roles in various image processing applications. Basically there are two types of objective quality or distortion assessment approaches. The first is mathematically defined measures such as Mean Square Error (MSE), Root Mean Square Error (RMSE) and Peak Signal-Noise Ratio (PSNR). The second considers Human Visual System (HVS) characteristics in an attempt to incorporate perceptual quality measures.

In practice, however, the HVS is more tolerant to a certain amount of noise than to a reduced sharpness. Moreover the visual quality is highly subjective and difficult to express objectively. In addition, the HVS is also highly in tolerant artefacts like "blips" and "bumps" on the reconstructed image. In the absence of accurate mathematical model for the complete HVS there is no reliable standard measure of image quality that is consistent with human perception and that provides qualitative as well as quantitative measurements. In spite of the lack of such an ideal measure, there are "acceptable" image quality measures that have been consistently used in the literature. One commonly used image quality measure is known as Root Mean Square Error (RMSE). Although it does not always correlate with human perception it is considered as "good" measure of fidelity of an image estimate.

\section{SOURCES OF NOISE}

Researchers are mainly concerned with the noise in a transmission system; usually the transmission channel is linear but dispersive due to a limited bandwidth. The image signal may be transmitted either in the analog form or in digital form. When an analog image signal is transmitted through a linear dispersive channel, the image edges get blurred and image signal gets contaminated with AWGN since no channel is noise free. The noise introduced in the transmission channel of a communication system will be considered in analog form. If the channel is so poor that the noise variances is high enough and make the signal excursive to very high positive or high negative value, the thres holding operation which is done at the front end of the receiver will contribute to saturated maximum and minimum values. Such noisy pixels will be seen as white and black spots. Therefore this type of noise is known as Salt and Pepper Noise (SPN). If analog image signal is transmitted the signal gets corrupted with AWGN and SPN as well. Thus there is an effect of mixed noise. If the image signal is transmitted in digital form through a linear dispersive channel, then a noise is introduced due to Bit Error called Inter Symbol Interference (ISI) which takes place along with AGWN which makes the situation worse. Due to ISI and AWGN, it may happen that 1 may be recognized as 0 and vice versa. Under such circumstance, the image pixel values have changed to some random values at random positions in the image frame. Such type of noise is known as RandomValued Impulse Noise (RVIN). Such kinds of error are taken care by the proposed Switching Weighted Adaptive Median (SWAM) Filter.

\section{DENOISING METHODS}

There are two basic approaches to image denoising, spatial domain filtering methods and transform domain filtering methods.

\section{Spatial Domain Filtering Methods:}

A traditional way to remove noise from image data is to employ spatial filters. Spatial filters are further classified into linear filters and non-linear filters. Linear filters process time-varying input signals to produce output signals, subject to the constraint of linearity. These results from systems composed solely of components (or digital algorithms) classified as having a linear response. In signal processing, a nonlinear (or non-linear) filter is a filter whose output is not a linear function of its input. That is, if the filter outputs signals $\mathrm{R}$ and $\mathrm{S}$ for two input signals $r$ and s separately, but does not always output $\alpha \mathrm{R}+\beta \mathrm{S}$ when the input is a linear combination $\alpha \mathrm{r}+$ $\beta$ s.

\section{Transform Domain Filtering Methods:}

The Transform Domain Filtering methods can be classified according to the choice of the basis or analysis function. The analysis functions can be further classified as Spatial Frequency Filtering and Wavelet domain 
Spatial Frequency Filtering refers to low pass filters using Fast Fourier Transform (FFT). In frequency smoothing methods the removal of the noise is achieved by designing a frequency domain filter and adapting a cut-off frequency to distinguish the noise components from the useful signal in the frequency domain. These methods are time consuming and depend on the cut-off frequency and the filter function behaviour. Furthermore they may produce frequency artefacts in the processed image. Noise is usually concentrated in high frequency components of the signal which corresponds to small detail size when performing a wavelet analysis. Therefore removing some high frequency (small detail components) which may be distorted by noise is adenoising process in the wavelet domain. Filtering operations in wavelet domain can be categorized in to wavelet thres holding, statistical wavelet coefficient model and undecimated. Wavelet domain transform based methods

\section{CONCLUSION:}

In this paper I have briefly described image processing concept with image nosining. Image denoising is a process to remove the noise that gets captured during image accusation process. The paper describes basic fundamentals of image denoising.

\section{REFERENCES:}

1. Achim A. and Kuruoglu E. E. (2005), "Image Denoising using Bivariate alpha-Stable Distributions in the Complex Wavelet Domain", IEEE Signal Processing Letters, 12, pp. 17-20.

2. Ahmed N., Natarajan T. and Rao K.R. (1974), "Discrete Cosine Transform" IEEE Transactions on Computers, Vol. C-23, No. 1, pp. 90-93.

3. Alajlan N., Kamel M. and Jernigan M. E. (2004), "Detail preserving impulsive noise removal,
Signal Processing", Image Communication, 19(10), 993-1003.

4. Andrew Bruce, David Donoho and Hung-YeGao (1996), "Wavelet Analysis", IEEE Spectrum, pp.27-35.

5. Arce G. and Paredes J. (2000), "Recursive Weighted Median Filters Admitting Negative Weights and their Optimization", IEEE Tr. on Signal Proc., Vol.48, No. 3.

6. Archibald R. and Gelb A. (2002), "Reducing the effects of noise in MRI reconstruction", pp. 497500 .

7. Arivazhagan. S., Deivalakshmi S. and Kannan. K. (2006), "Technical Report on Multi-resolution Algorithms for Image Denoising and Edge Enhancement for CT images using Discrete Wavelet Transform".

8. Arivazhagan S., Deivalakshmi S., Kannan K., Gajbhiye B. N., Muralidhar C., SijoN Lukose and Subramanian M.P. (2007), "Performance Analysis of Wavelet Filters for Image Denoising", Advances in Computational Sciences and Technology, Vol.1 No. 1, pp. 1-10.

9. Barten P. G. J. (1999), "Contrast sensitivity of the human eye and its effects on image quality", SPIE Optical Engineering Press, Bellingham, WA.

10. Black M., Sapiro G. Marimont D. and Heeger D. (1998), "Robust Anisotropic Diffusion", IEEE Trans. Image Processing, 7, pp.421-432.

11. Blum R.S. and Liu Z. (2006), "Multi-Sensor Image Fusion and Its Applications", Boca Raton: Taylor \& Francis.

12. Brownrigg D. R. K. (1984), "The weighted median filter," Common. ACM, Vol. 27, No. 8, pp. 807-818. 